\section{Pandemia por COVID-19 no Brasil: análise da demanda e da oferta de leitos hospitalares e equipamentos de ventilação assistida segundo diferentes cenários}

\author{
The COVID-19 pandemic in Brazil: analysis of \\ supply and demand of hospital and ICU beds and \\ mechanical ventilators under different scenarios
}

\author{
Pandemia por COVID-19 en Brasil: análisis de la \\ demanda y oferta de camas hospitalarias, así \\ como equipamientos de ventilación asistida, \\ según diferentes escenarios
}

Kenya Valeria Micaela de Souza Noronha 1

Gilvan Ramalho Guedes 1

Cássio Maldonado Turra 1

Mônica Viegas Andrade 1

Laura Botega 1

Daniel Nogueira 1

Julia Almeida Calazans 1

Lucas Carvalho 1

Luciana Servo 2

Monique Félix Ferreira 1,3

doi: 10.1590/0102-311X00115320

\section{Resumo}

O objetivo deste estudo é analisar a pressão sobre o sistema de saúde no Brasil decorrente da demanda adicional gerada pela COVID-19. Para tanto, foi realizado um conjunto de simulações para estimar a demanda de leitos gerais (microrregiões de saúde), leitos de UTI e equipamentos de ventilação assistida (macrorregiões de saúde) em diferentes cenários, para intensidade (taxas de infecção equivalentes a 0,01, 0,1 e 1 caso por 100 habitantes) e horizontes temporais (1, 3 e 6 meses). Os resultados evidenciam uma situação crítica do sistema para atender essa demanda potencial, uma vez que diversas microrregiões e macrorregiões de saúde operariam além de sua capacidade, comprometendo $o$ atendimento a pacientes principalmente aqueles com sintomas mais severos. O estudo apresenta três mensagens relevantes. Em primeiro lugar, é necessário reduzir a velocidade de propagação da COVID-19 na população brasileira, permitindo um tempo maior para a reorganização da oferta e aliviando a pressão sobre o sistema de saúde. Segundo, é necessário expandir o número de leitos disponiveis. Ainda que o setor privado contribua para amortecer o déficit de demanda, a oferta conjunta dos dois setores não seria suficiente em várias macrorregiões. A construção de hospitais de campanha é importante, tanto em locais onde historicamente há vazios assistenciais como também naqueles onde já se observa uma pressão do lado da demanda. A terceira mensagem diz respeito à organização regionalizada dos serviços de saúde que, apesar de adequada em situações de demanda usual, em momentos de pandemia este desenho implica desafios adicionais, especialmente se a distância que o paciente tiver de percorrer for muito grande.

COVID-19; Pandemias; Acesso aos Serviços de Saúde; Sistema Único de Saúde; Saúde Suplementar
Correspondência

K. V. M. S. Noronha

Centro de Desenvolvimento e Planejamento Regional, Faculdade de Ciências Econômicas, Universidade Federal de Minas Gerais. Av. Presidente Antônio Carlos 6627, Belo Horizonte, $M G$ 31270-901, Brasil.

knoronha@cedeplar.ufmg.br

1 Faculdade de Ciências Econômicas, Universidade Federal de Minas Gerais, Belo Horizonte, Brasil.

2 Diretoria de Estudos e Políticas Sociais, Instituto de Pesquisa Econômica Aplicada, Brasília, Brasil.

3 Secretaria de Estado de Saúde de Minas Gerais, Belo Horizonte, Brasil. 


\section{Introdução}

Desde que foi detectada em dezembro de 2019, a COVID-19 vem se alastrando pelos diferentes continentes, tendo sido caracterizada como uma pandemia pela Organização Mundial da Saúde (OMS). Segundo a OMS, 80\% dos pacientes com COVID-19 apresentam sintomas leves e sem complicações, $15 \%$ evoluem para hospitalização que necessita de oxigenoterapia e 5\% precisam ser atendidos em unidade de terapia intensiva (UTI) 1. Dependendo da velocidade de propagação do vírus na população, os sistemas de saúde podem sofrer forte pressão decorrente da demanda extra gerada pela COVID-19.

Vários estudos têm sido conduzidos para avaliar a capacidade de atendimento hospitalar em diversos países. Uma das principais conclusões é que se nenhuma ação de ampliação da oferta de leitos ou de contenção do vírus for realizada, haverá alta probabilidade de saturação dos sistemas de saúde em um espaço de tempo relativamente curto 2,3,4,5,6,7,8. A demanda por hospitalizações entre os pacientes de COVID-19 é influenciada por diferentes fatores, tais como idade e condições preexistentes, com efeitos distintos nas sociedades em que a pandemia avança 2,3,4,5,6,7,9,10,11.

Para o Brasil, estudos sobre o tema também têm sido desenvolvidos, tanto com abrangência nacional 12,13,14 como regional 15,16,17,18. Castro et al. 12 estimaram a data de colapso do sistema de saúde nas macrorregiões onde estão localizadas diversas capitais brasileiras. A precisão das datas apresentadas, no entanto, é suscetível aos vários pressupostos adotados nas diferentes simulações. Rache et al. 13,14 apontaram as regiões de saúde com maior vulnerabilidade à pandemia da COVID-19, assim como a necessidade de leitos de UTI. As estimativas de oferta foram realizadas considerando-se apenas leitos do Sistema Único de Saúde (SUS) e não levaram em consideração fatores demográficos associados à variação regional dos casos.

O objetivo do presente artigo é analisar a pressão sobre o sistema de saúde no Brasil decorrente da demanda adicional gerada pela COVID-19. Os serviços analisados são leitos gerais, de UTI e equipamentos de ventilação assistida. Dois conjuntos de simulações são realizados, um com a oferta conjunta SUS e privada, e outro apenas com a oferta SUS. As estimativas consideram as diferenças no perfil etário em relação às taxas de infecção e de internação. Diferentes cenários para as taxas de infecção e horizontes temporais foram definidos devido ao desconhecimento sobre a verdadeira trajetória da COVID-19 no território nacional. Como o Brasil é um país de grandes dimensões, com presença de áreas remotas, as desigualdades de acesso geográfico podem significar barreiras fundamentais para a obtenção do cuidado no cenário pandêmico. Para os casos de hospitalização, nem sempre os atendimentos serão realizados no próprio município de residência, exigindo o encaminhamento destes pacientes. Dessa forma, foi também analisada a dificuldade de acesso para conseguir o atendimento, com base na distância média mínima percorrida entre o município de residência do paciente e o município mais próximo com hospital com leito de UTI.

\section{Metodologia}

O número de internações hospitalares foi estimado para as microrregiões (leitos gerais) e macrorregiões (leitos de UTI e aparelhos de ventilação mecânica) de saúde, segundo diferentes cenários de intensidade e duração da infecção por COVID-19. A regionalização foi definida baseando-se na classificação de 2019, na qual os municípios são divididos em 450 microrregiões e 118 macrorregiões de saúde, elaborada valendo-se dos Planos Diretores de Regionalização dos estados (PDR). Excluímos o Distrito Federal devido à sua peculiaridade político-administrativa.

Para o cálculo da demanda, foram usadas três funções distintas: (1) a população estimada para o ano de 2020 por grupos de idade e região de saúde; (2) distribuição proporcional por idade das taxas de infecção confirmadas por COVID-19; e (3) taxas específicas por grupo de idade de internação hospitalar geral e UTI por COVID-19. Utilizamos as estimativas populacionais por idade e município produzidas por Freire 19.

O número esperado de casos confirmados por idade foi calculado pela multiplicação das taxas de infecção e a proporção da população, ambas por grupo de idade, em cada localidade de análise. Posteriormente, normalizamos os valores obtidos de forma a totalizarem taxas de infecção equivalentes 
a $0,01 \%, 0,1 \%$ e $1 \%$ da população em cada região. Na etapa seguinte, calculamos o número total de internações em cada grupo etário ao multiplicar o número esperado de casos pelas taxas específicas por grupo de idade de internação geral ou UTI. Neste estudo, supomos que a demanda estimada por aparelhos de ventilação mecânica é igual à demanda estimada por leitos de UTI.

Como a cobertura dos testes de COVID-19 na população brasileira é ainda baixa, as informações sobre os casos confirmados da doença, internação hospitalar geral e de UTI por grupos de idade podem ser pouco confiáveis para serem utilizadas em simulações. Portanto, neste artigo, adotamos como padrão taxas específicas por idade estimadas para os Estados Unidos 9 e para a Espanha 20.

Utilizamos dois bancos de dados oficiais: Sistema de Informações Hospitalares do SUS (SIH/SUS) e Cadastro Nacional de Estabelecimentos de Saúde (CNES). As informações de internações hospitalares referem-se às realizadas pelo SUS em todo o ano de 2019, já as de oferta (CNES), aos registros do mês de dezembro deste mesmo ano. Foram excluídos os leitos obstétricos, pediátricos, hospital/ dia e especiais (no caso de leitos gerais) e leitos pediátricos, neonatais e queimados (no caso de leitos de UTI). A exclusão dos leitos pediátricos e neonatais decorre da impossibilidade de atendimento da população adulta nestes leitos. Como a taxa de infecção e de internação por COVID-19 em crianças e adolescentes é muito baixa, a inclusão desses leitos geraria uma superestimação da oferta disponível.

Para cada um dos serviços $i$ analisados (leitos gerais, de UTI e aparelhos de ventilação mecânica), calculou-se a taxa de ocupação em cada um dos cenários de tempo $m$ analisados (Equação 1).

$$
\operatorname{Txo}_{i}^{m}=\frac{P_{i}^{m}}{D_{i}^{m}}
$$

Em que: $D_{i}^{m}=$ número de dias disponíveis de cada serviço $i$ no período $m$, com $m=1,3$ ou 6 meses; $P_{i}^{m}=$ número de dias de permanência em cada serviço $i$ e cenário de tempo $m$. Para o caso de aparelhos de ventilação mecânica, considerou-se o número de dias de permanência em UTI.

Para conhecer a demanda extra gerada pela COVID-19, calculou-se o total de dias de permanência em cada um dos cenários de taxa de infecção $\left(P_{i}^{T I, m}\right)$, que adicionado ao total de dias de permanência observado em 2019 com base nas informações do SIH/SUS $\left(P_{i}^{m}\right)$, resultou no total simulado de dias de permanência $\left(T P_{i}^{T I, m}\right)$ :

$$
T P_{i}^{T I, m}=P_{i}^{T I, m}+P_{i}^{m}
$$

Em que: $T I=$ taxa de infecção definida por cada um dos cenários $(0,01 \%, 0,1 \%$ e $1 \%) ; P_{i}^{T I, m}=$ total de casos da COVID-19 em cada cenário, multiplicado pelo tempo médio de permanência hospitalar segundo o nível de agravo -8 dias para casos mais leves e 10 dias para casos mais graves 21 .

As estimativas da demanda total $\left(P_{i}^{T I, m}\right)$ por cada um dos serviços analisados $i$, em cada um dos cenários de tempo $(m)$ e de infecção (TI), foram obtidas da seguinte forma:

$$
D T_{i}^{T I, m}=\frac{T P_{i}^{T L, m}}{D_{i}^{m}}
$$

Notem que além das internações por COVID-19, foram consideradas as demais causas de internação como fonte de ocupação dos leitos. Devido à indisponibilidade de informações, consideramos a taxa de ocupação dos leitos privados como sendo igual à média de cada micro/macrorregião estimada para os leitos SUS em 2019. Para entender a importância do setor privado na ampliação da oferta desses serviços, as simulações foram também realizadas excluindo-se os leitos deste setor. Um exercício adicional foi realizado considerando-se as taxas de infecção totais observadas no dia 4 de maio de 2020 em cada micro/macrorregião de saúde no Brasil, supondo que elas foram alcançadas em três cenários de tempo: 1, 3 e 6 meses. Os casos foram ajustados levando-se em conta um nível de cobertura dos testes de diagnóstico igual a $13 \%$ 22. Os procedimentos metodológicos para estimar as taxas de internação por idade em cada localidade foram os mesmos usados para as simulações realizadas no presente artigo.

Além das simulações, caracterizamos a oferta dos serviços hospitalares no Brasil, considerando o total de hospitais e distinguindo por natureza jurídica. Como o atendimento de alta complexidade é organizado no nível macrorregional, analisamos também a distância mínima que os indivíduos 
precisam percorrer para obter atendimento fora de seu município de residência. Para esse cálculo, foi utilizada a informação do município de residência do paciente e de localização do hospital com leito de UTI e o menor caminho a ser percorrido em transporte multimodal 23.

\section{Resultados}

\section{Caracterização da oferta}

Em 2019, o Brasil apresentava 8.139 estabelecimentos hospitalares e 490.397 leitos. Essa oferta equivale a aproximadamente 2,3 leitos por 1.000 habitantes, o que corresponde a praticamente metade da média observada em 2017 para os países da Organização para a Cooperação e Desenvolvimento Econômico (OCDE) 24. Ainda que distante da média da OCDE, a oferta de leitos totais no Brasil é equiparada a de países como Canadá, Reino Unido e Suécia, o que sugere que nossa oferta é relativamente condizente à de países com sistemas de saúde majoritariamente públicos e bem organizados. Uma diferença importante diz respeito à segmentação do sistema brasileiro, que tem consequências na composição público-privada no cuidado hospitalar.

O Brasil conta com 270.880 leitos gerais (clínicos e cirúrgicos) e 34.464 leitos de UTI adultos, sendo $66 \%$ e $48 \%$ disponíveis para o SUS, respectivamente. Chama a atenção o elevado número de hospitais de pequeno porte, 5.345 hospitais (66\%), dos quais 70\% têm até 29 leitos. Somente 10\% dos estabelecimentos hospitalares são de grande porte (acima de 150). Embora em menor número, esses hospitais concentram $42 \%$ dos leitos, seguidos dos de médio porte (51 a 150 leitos), com 35\%. A taxa de ocupação dos leitos gerais no SUS é relativamente baixa para os hospitais de pequeno porte, $24 \%$ (até 29 leitos) e 32\% (entre 30 e 50 leitos), comparada a 75\% nos hospitais de grande porte. Para leitos de UTI, percebe-se o esgotamento maior do sistema de saúde, principalmente, nos hospitais de grande porte, com taxa de ocupação média de $60 \%$ (médio porte) e $77 \%$ (grande porte).

A Figura 1 apresenta a distribuição da oferta de leitos gerais, de UTI e aparelhos de ventilação mecânica por 10 mil habitantes disponíveis no Brasil em 2019 por micro/macrorregião de saúde. Analisando o total de leitos gerais, a oferta é verificada em todas as 449 microrregiões, mas com dispersão espacial importante (Figura 1a). A média por 10 mil habitantes é de 12,2, variando de 2,0 (em Sergipe) a 30,3 (em Goiás). As microrregiões com menor oferta estão concentradas no Amazonas, Pará, Roraima, Minas Gerais e Ceará. A população dessas microrregiões chega a representar 26\% da população do Ceará, 22\% do Amazonas e 20\% do Pará. As microrregiões com oferta mais elevada estão localizadas principalmente no Rio Grande do Sul, Santa Catarina, São Paulo, Mato Grosso e Goiás.

Considerando somente os leitos gerais SUS (Figura 1b), a média por 10 mil habitantes cai para 9,1. Embora existam leitos SUS em todas as microrregiões, esta oferta também é desigual no território: $25 \%$ das microrregiões com menor oferta têm menos de 6,6 leitos por 10 mil habitantes, contrapondo com uma oferta superior a 11 entre aquelas do maior quartil da distribuição. Em Rondônia, Roraima, Piauí, Minas Gerais e Rio Grande do Sul, mais de 50\% da população total estão em microrregiões com elevada oferta de leitos SUS.

A oferta privada é nula em 36 microrregiões e varia de 0,03 leitos por 10 mil habitantes em São José de Mipibu (Rio Grande do Norte) a 11,5 em São José do Rio Preto (São Paulo) (Figura 1c). A participação desse setor é mais elevada em microrregiões de São Paulo, Paraná, Rio Grande do Sul, Minas Gerais e Goiás.

A oferta de leitos de UTI está presente em todas as macrorregiões brasileiras, exceto na Litoral Leste/Jaguaribe no Ceará que tem oferta nula (Figura 1d). Entre as macrorregiões com oferta positiva, a distribuição varia de 0,07 (Oeste no Amazonas) a 3,3 (RRAS6 em São Paulo). As macrorregiões com menor oferta estão em sua maioria no Norte e Nordeste do país. Em contraposição, as 30 macrorregiões que apresentam maior oferta estão em São Paulo, Paraná, Santa Catarina, Rio Grande do Sul, Mato Grosso do Sul, Mato Grosso e Goiás. Há uma complementariedade dos setores público e privado na oferta de leitos de UTI. A participação privada só é nula em oito macrorregiões localizadas principalmente nos estados do Norte (Figura 1f). Em seis macrorregiões, a oferta privada responde por mais de 70\% da oferta de leitos de UTI: Centro-Norte (Mato Grosso), Macrorregião II (Rio de Janeiro), Norte (Espírito Santo), Nordeste (Goiás), Semiárido (Piauí) e Sertão Central (Ceará). 


\section{Figura 1}

Distribuição da oferta de leitos gerais e de unidade de terapia intensiva (UTI) e aparelhos de ventilação mecânica. Brasil, 2019.

1a) Leitos gerais (microrregiões) - Total

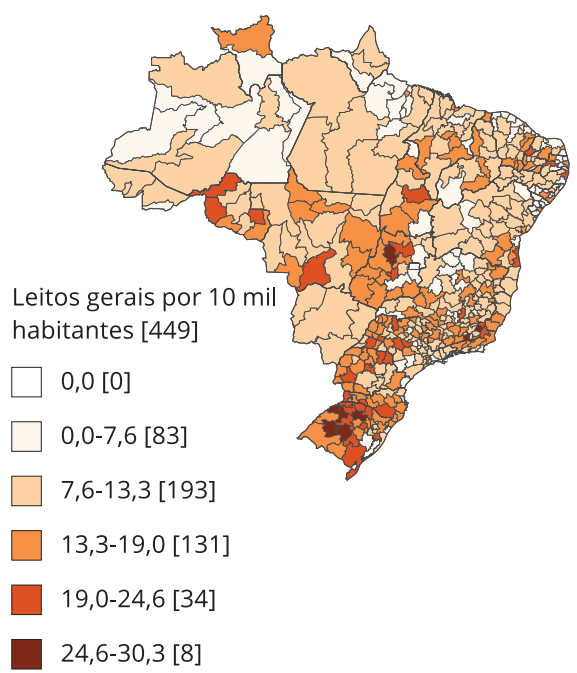

1d) Leitos de UTI (macrorregiões) - Total

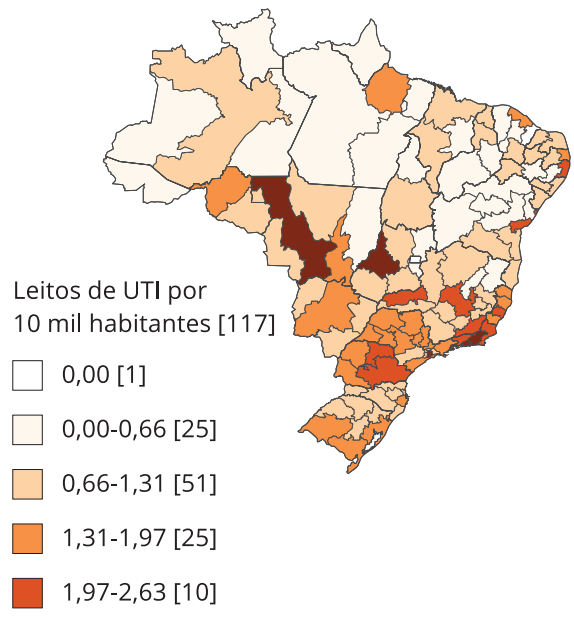

$2,63-3,28[5]$ 1b) Leitos gerais (microrregiões) - SUS

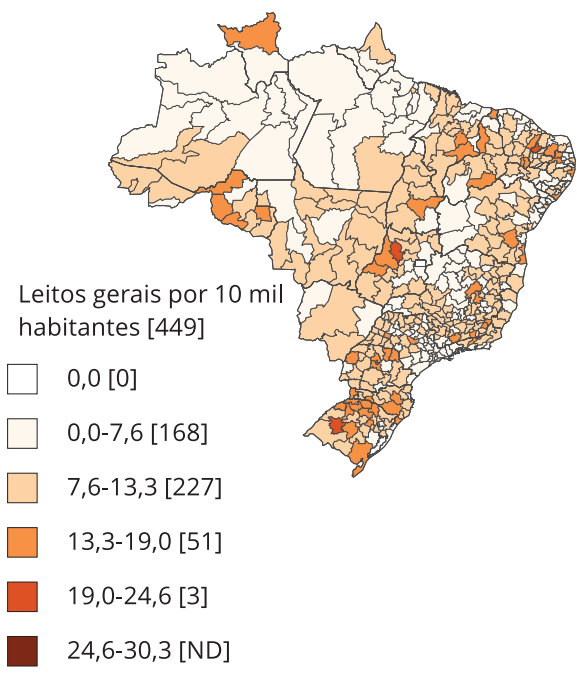

1e) Leitos de UTI (macrorregiões) - SUS

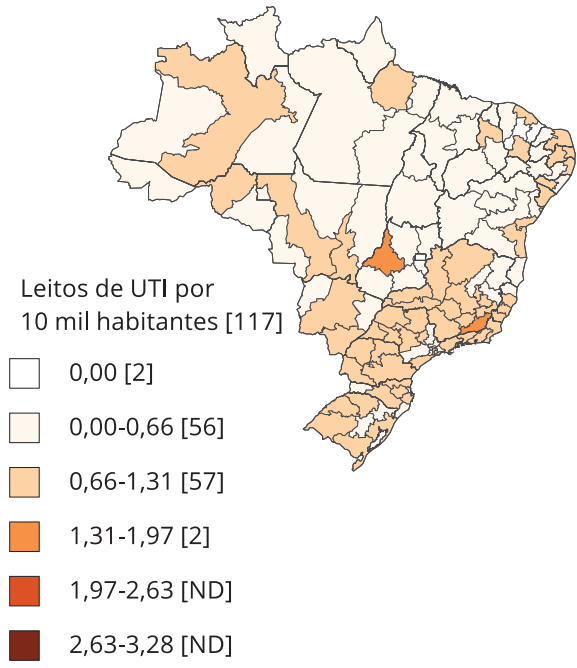

1c) Leitos gerais (microrregiões) - Privados

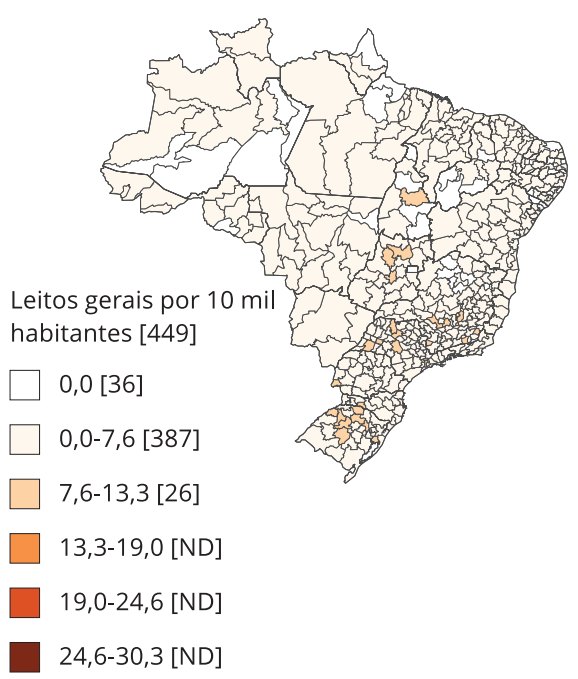

1f) Leitos de UTI (macrorregiões) - Privados

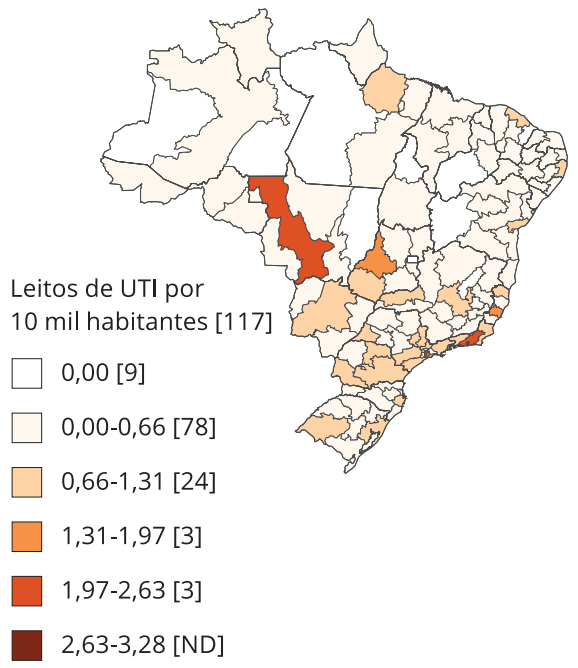

(continua) 
1g) Aparelhos de ventilação mecânica (macrorregiões) - Total

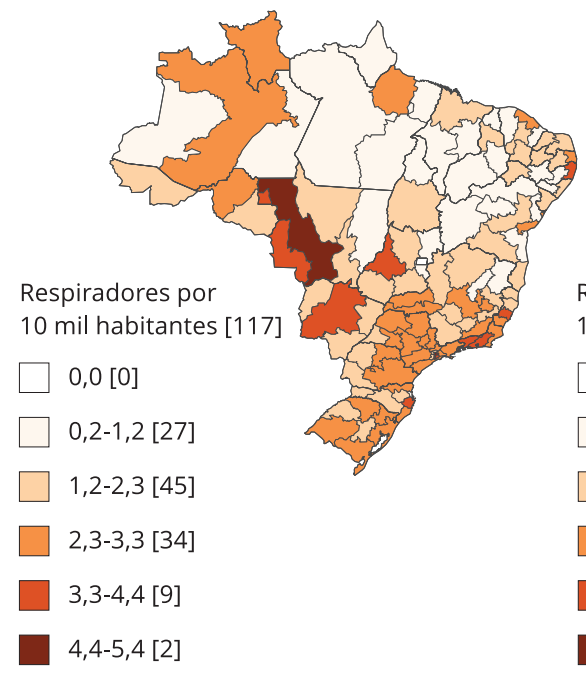

1h) Aparelhos de ventilação mecânica (macrorregiões) - SUS

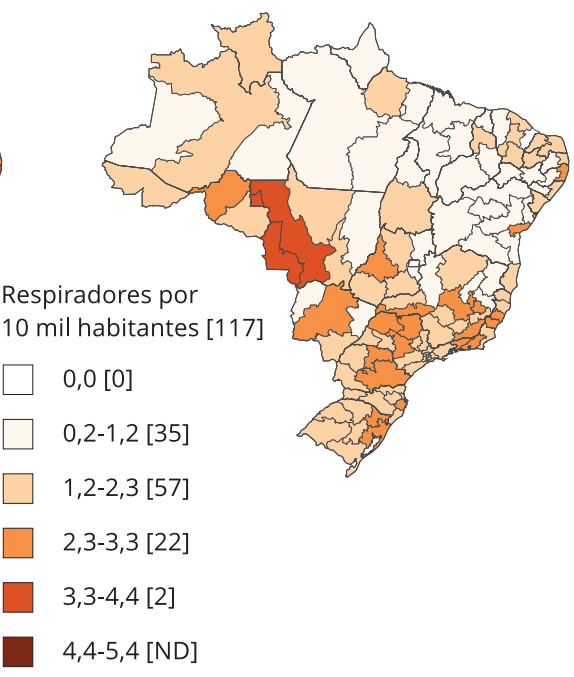

1i) Aparelhos de ventilação mecânica (macrorregiões) - Privados

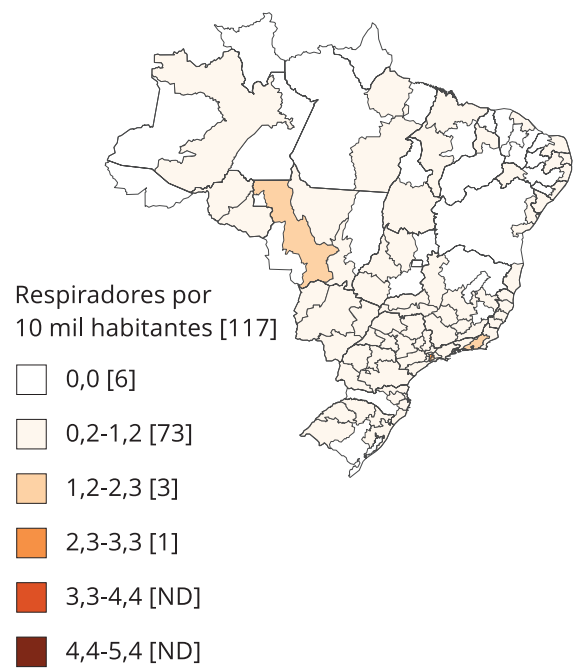

ND: não disponível.

A distribuição da oferta de aparelhos de ventilação mecânica entre as macrorregiões de saúde é apresentada nas Figuras 1g, 1h e 1i. Em 2019, o país contava com 57.303 aparelhos, sendo $72 \%$ disponíveis para o SUS. A média de respiradores entre as macrorregiões é de 2,0 por 10 mil habitantes, levando-se em conta toda a oferta, e 1,65 se incluirmos somente os disponíveis para o SUS. A oferta pública (Figura 1h) é verificada em todas as macrorregiões de saúde, mas apresenta grande dispersão no território. A menor taxa é observada na macrorregião Litoral Leste/Jaguaribe (Ceará), com oferta ínfima de 0,07 respiradores por 10 mil habitantes, sendo a única sem leitos de UTI. As macrorregiões com oferta inferior a 0,5 aparelhos por 10 mil habitantes são as mesmas que apresentam infraestrutura de leitos de UTI insuficiente. As que apresentam oferta SUS mais elevada são a Norte e Sul no Mato Grosso, Metropolitana no Espírito Santo e Grande Florianópolis em Santa Catarina. A participação privada na oferta de respiradores varia de 0 a 55\%, sendo maior em São Paulo, Mato Grosso e Rio de Janeiro (Figura 1i).

A Figura 2a apresenta a menor distância média que os residentes precisariam percorrer para conseguir atendimento em leito de UTI na macrorregião de residência. A distância mínima a ser percorrida é relativamente baixa, $98 \mathrm{~km}$ em média, equivalente a cerca de uma hora e meia para o deslocamento. A distribuição dessa distância, entretanto, é bastante desigual entre as grandes regiões, sendo maior no Norte, Nordeste e Centro-oeste. Entre os estados, chama a atenção o Amazonas com distância média de $615 \mathrm{~km}$, seguido do Amapá $(259 \mathrm{~km})$. As dificuldades de acesso podem estar presentes em $8 \%$ dos municípios brasileiros, nos quais os pacientes precisariam percorrer uma distância superior a 240km. Dentre esses municípios, 126 estão no Norte (28\% dos municípios da região), 165 no Nordeste (9\%), 63 no Sudeste (4\%), 102 no Centro-oeste (22\%) e 8 no Sul (0,7\%). Essas macrorregiões são exatamente aquelas que apresentam densidade demográfica baixa (Figura 2b). 
2a)

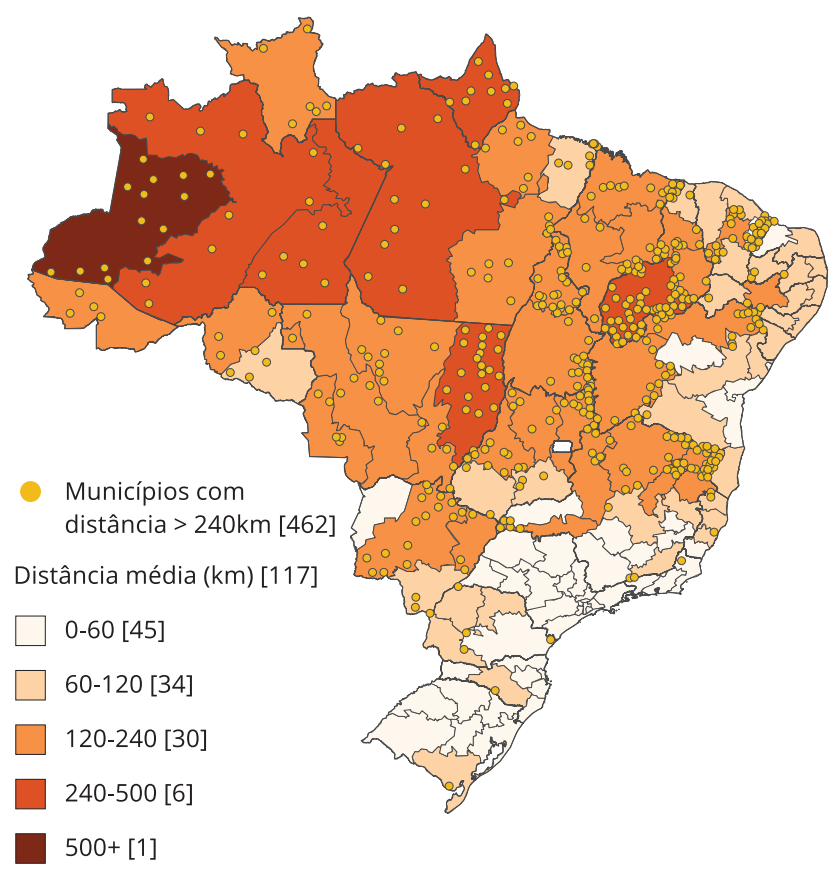

2b)

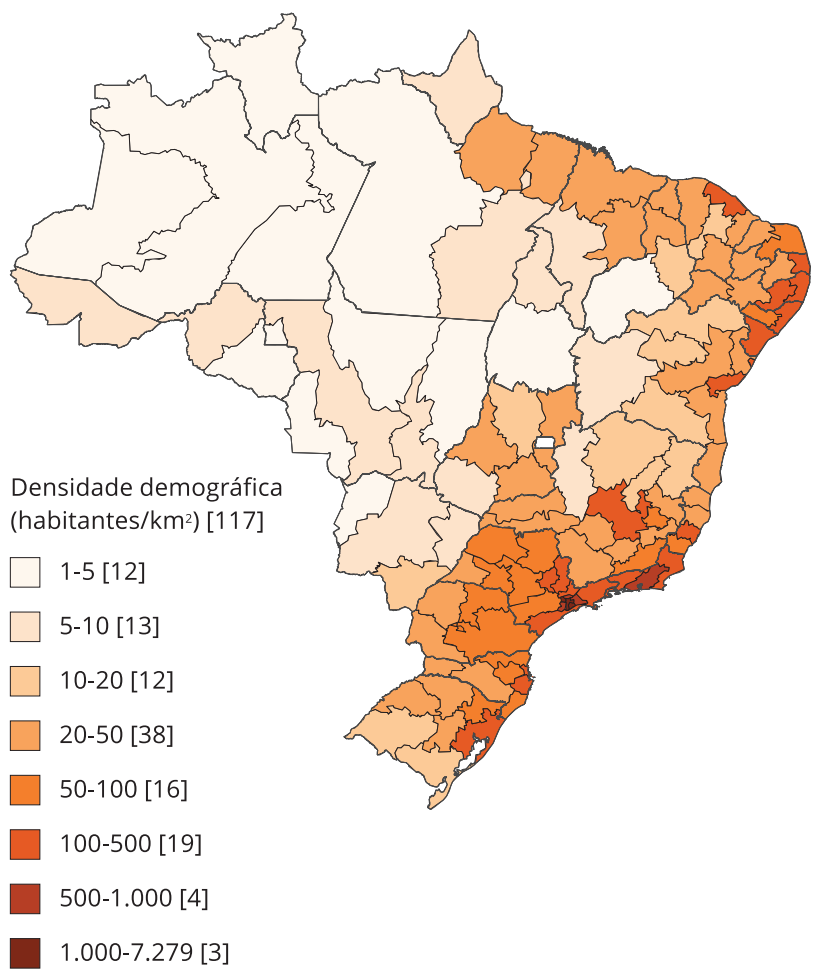

\section{Pressão da demanda extra gerada pela COVID-19}

Os resultados apresentados nesta seção foram estimados com base nas distribuições etárias de infecção e hospitalização norte-americanas. Os resultados com os parâmetros espanhóis são apresentados no Material Suplementar (http://cadernos.ensp.fiocruz.br/static/arquivo/supple00115320-pt_4059.pdf). O padrão internacional escolhido pouco influencia as distribuições regionais e a demanda, principalmente para leitos de UTI e aparelhos de ventilação mecânica, mantendo as conclusões gerais independentemente do padrão adotado.

\section{- Leitos gerais}

Considerando-se a oferta privada e a do SUS, os principais problemas começariam a surgir quando a taxa de infecção alcançasse $1 \%$ da população. Para taxas menores, $0,01 \%$ e $0,1 \%$, independentemente do horizonte temporal, praticamente todas as 449 microrregiões estariam operando com níveis inferiores à sua capacidade (resultados disponíveis, mas não apresentados). Se a taxa de infecção por COVID-19 atingir 1\% em 1 mês, 136 microrregiões de saúde no Brasil (30\%) estariam com sua capacidade comprometida (Figuras 3a, 3b e 3c).

Excluindo os leitos privados, o quadro se tornaria mais grave. A uma taxa de infecção de $1 \% \mathrm{em} 1$ mês, o colapso do sistema seria observado em todas as grandes regiões (Figuras 3d, 3e e 3f). No norte do Brasil, do total de 45 microrregiões, $49 \%$ teriam a capacidade de atendimento comprometida se 


\section{Figura 3}

Percentual de leitos gerais e de unidade de terapia intensiva (UTI) ocupados em cada micro/macrorregião de saúde, considerando taxas de infecção por COVID-19 igual a 1\% da população e três horizontes temporais: 6 meses, 3 meses e 1 mês. Brasil.

3a) Oferta SUS e privada - leitos gerais (microrregião) - 6 meses

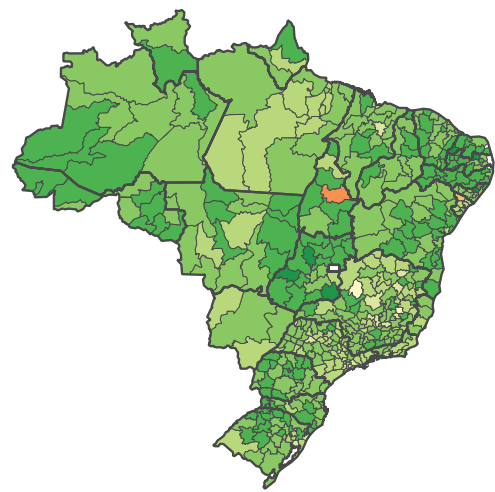

3d) Apenas oferta SUS - leitos gerais (microrregião) - 6 meses

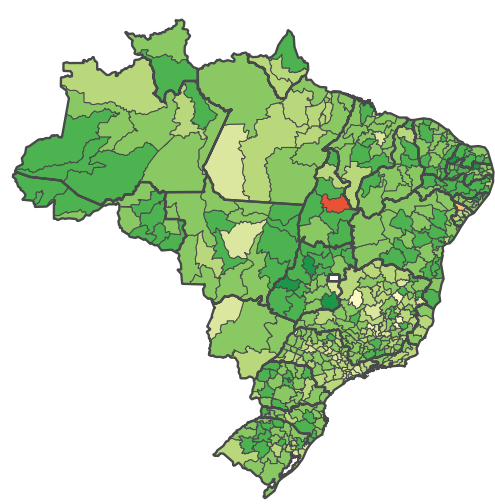

3g) Oferta SUS e privada - leitos de UTI (macrorregião) - 6 meses

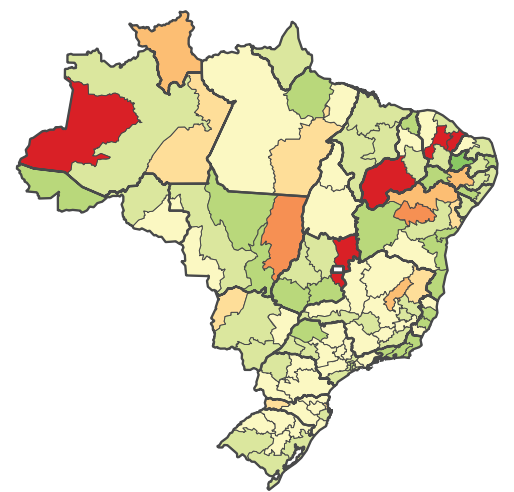

3b) Oferta SUS e privada - leitos gerais (microrregião) - 3 meses

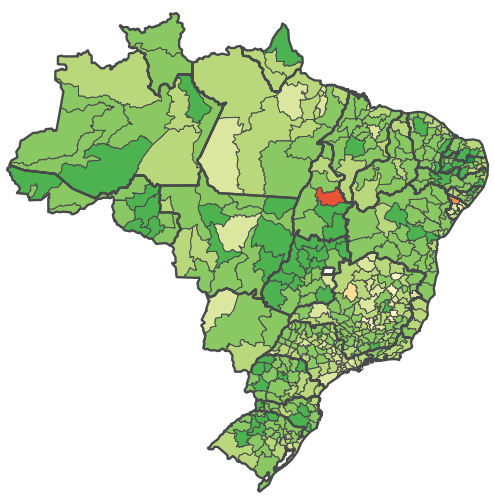

3e) Apenas oferta SUS - leitos gerais (microrregião) - 3 meses

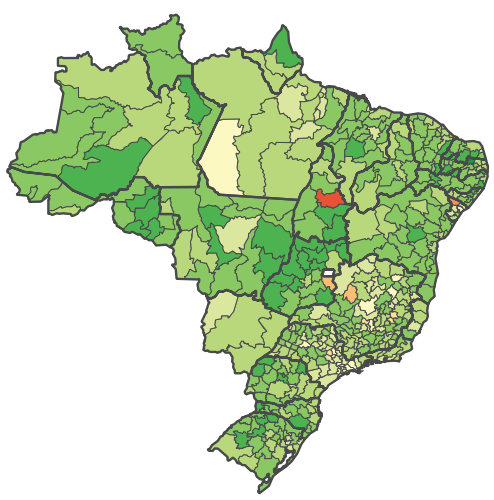

3h) Oferta SUS e privada - leitos de UT (macrorregião) - 3 meses

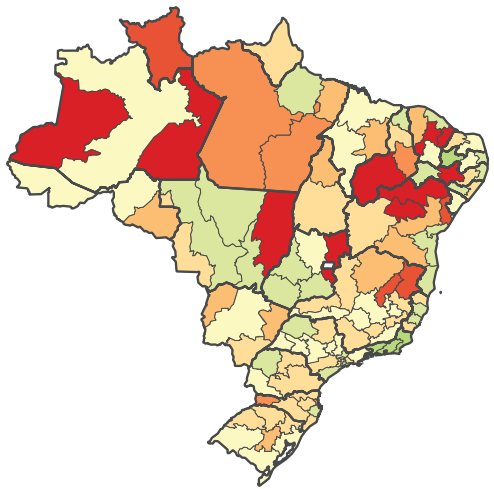

3c) Oferta SUS e privada - leitos gerais (microrregião) - 1 mês

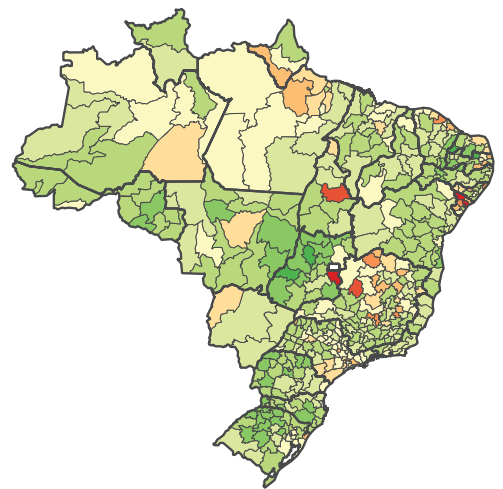

$0-20$

$20-40$

$40-60$

$60-80$

80-100

$100-120$

$120-140$

$140-160$

$160-180$

$180-200$

3f) Apenas oferta SUS - leitos gerais (microrregião) - 1 mês

$200+$

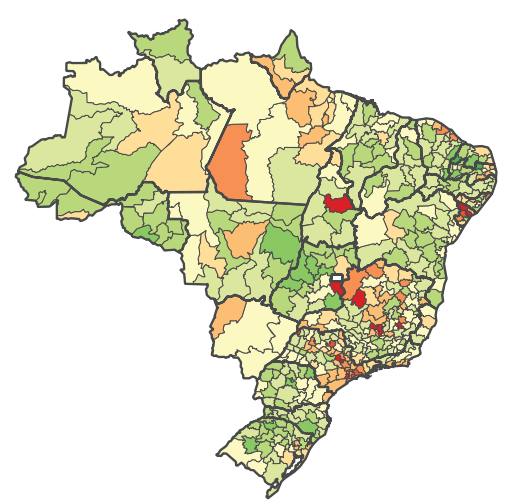

3i) Oferta SUS e privada - leitos de UTI (macrorregião) - 1 mês

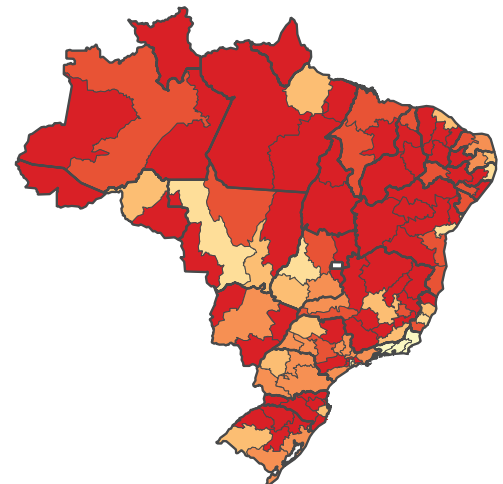

(continua) 
3j) Apenas oferta SUS - leitos de UTI (macrorregião) -6 meses

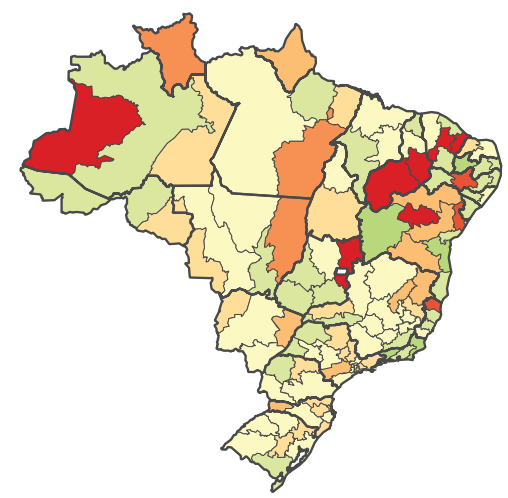

3k) Apenas oferta SUS - leitos de UTI (macrorregião) - 3 meses

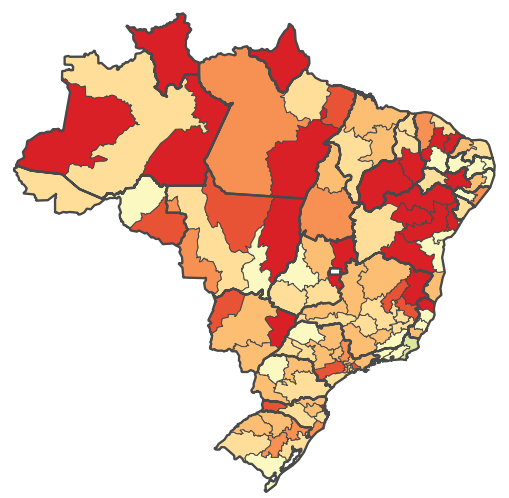

3I) Apenas oferta SUS - leitos de UTI (macrorregião) - 1 mês

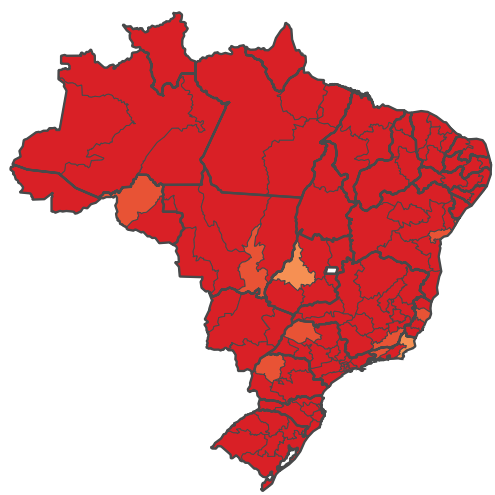

apenas os leitos SUS fossem considerados. Ao incorporar os leitos privados, esse percentual reduziria, mas ainda assim uma quantidade importante de microrregiões (38\%) teria dificuldade de atender toda a demanda. No Nordeste, quando apenas os leitos SUS são considerados, $34 \%$ das microrregiões apresentariam comprometimento da oferta no cenário analisado. Esse número reduziria para $26 \%$ se a oferta de leitos privados fosse incluída. No Sudeste, a importância do setor privado é ainda mais evidente. Considerando apenas a oferta do SUS, em torno de 67\% das microrregiões de saúde não teriam oferta suficiente para atender aos pacientes. A inclusão dos leitos gerais privados reduziria esse percentual para 42\%. No Sul e Centro-oeste do país, o comprometimento da oferta seria observado em 29\% e 32\% das microrregiões se apenas os leitos SUS fossem considerados. Esses percentuais reduziriam para $13 \%$ e $16 \%$, respectivamente, se o sistema puder contar com a oferta privada.

\section{- Leitos de UTI}

Os resultados da simulação evidenciam uma situação mais preocupante em relação a leitos de UTI. Esses resultados, contudo, dependem da velocidade de propagação da infecção em cada macrorregião de saúde. Em um cenário mais otimista (taxa de infecção de 0,01\% em 6 meses), contando com a oferta SUS e privada, seria observada uma sobrecarga em apenas 5 macrorregiões de saúde, sendo uma localizada em Minas Gerais (Triângulo do Norte), duas em Santa Catarina (Planalto Norte/Nordeste e Foz do Rio Itajaí) e duas no Ceará (Sertão Central e Litoral Leste/Jaguaribe) (resultados disponíveis, mas não apresentados). Em Sertão Central, a oferta de leitos de UTI ocorreria somente pelo setor privado (30 leitos), não havendo nenhum leito disponível para o SUS. A macrorregião Litoral Leste/Jaguaribe (Ceará) sequer tinha oferta de leito de UTI cadastrada em dezembro de 2019 no CNES.

Um cenário dramático seria observado se uma taxa de infecção mais elevada, de $1 \%$, for alcançada em 1 mês (Figuras 3g, 3h e 3i). Nesse caso, o sistema entraria em colapso na medida em que 100\% das macrorregiões estariam operando além de sua capacidade. Em 102 macrorregiões a demanda excedente estaria acima de 50\%, evidenciando ainda mais a fragilidade da oferta de leitos de UTI no país. As macrorregiões com os maiores déficits de oferta estariam localizadas no Amazonas, Goiás, Ceará, Piauí, Bahia, Pernambuco e Mato Grosso. Considerando apenas a oferta SUS, esse quadro se agravaria (Figuras 3j, 3k e 31). No cenário mais pessimista, a proporção de demanda extra não atendida seria menor se o sistema contar com a oferta de leitos privados. Nesse cenário, dois resultados merecem destaque, o da macrorregião RRAS6, que inclui o Município de São Paulo, e o da Macrorregião II, que inclui o Município do Rio de Janeiro. Sem os leitos privados, a demanda gerada pela COVID-19 
superaria a oferta disponível em 202\% e 245\%. Considerando a oferta SUS e privada, esse percentual seria bem menor, igual a $13 \%$ e $4,5 \%$, respectivamente.

\section{- Aparelhos de ventilação mecânica}

No caso dos aparelhos de ventilação mecânica, consideramos a oferta conjunta pública e privada. Apesar de ser possível identificar no CNES os aparelhos por natureza jurídica, esta informação pode não refletir exatamente a sua disponibilidade no setor público ou privado devido à forma como os dois setores interagem no sistema de saúde. Embora menos severos, os resultados também são preocupantes. No cenário mais pessimista, praticamente todo o sistema iria ao colapso, com $97 \%$ das macrorregiões apresentando comprometimento de sua capacidade de atendimento. Em 51 macrorregiões, o sistema de saúde não teria condições de atender a mais de $50 \%$ dos pacientes. O Nordeste (64\%) e o Norte (57\%) do país apresentariam a maior proporção de macrorregiões nessa situação mais crítica (Figura 4).

\section{Simulações com os níveis de infecção observados em cada micro/macrorregião de saúde}

Esta seção apresenta as simulações baseadas nas taxas de infecção observadas no dia 4 de maio de 2020 em cada localidade, com correção para sub-registro. O objetivo é avaliar o estágio atual da propagação e sua distribuição desigual no território nacional. Ao considerar a heterogeneidade da taxa de infecção entre regiões, estas simulações permitem controlar indiretamente por outros fatores que afetam a velocidade de propagação. Comparamos três cenários de tempo, levando-se em conta a oferta conjunta de leitos públicos e privados, para avaliar em que medida o achatamento da curva aliviaria o sistema de saúde. A análise mantém a oferta e taxa de ocupação dos leitos em níveis de 2019. Como o primeiro caso confirmado de COVID-19 no Brasil foi em 26 de fevereiro, o tempo que essas taxas foram alcançadas em cada localidade no país é inferior a 3 meses. No Brasil, até a data analisada, 6,7\% das microrregiões de saúde não tinham registros de infecção por COVID-19. Dentre

Figura 4

Percentual de aparelhos de ventilação mecânica ocupados por macrorregião de saúde, considerando taxas de infecção por COVID-19 igual a $1 \%$ da população e três horizontes temporais: 6 meses, 3 meses e 1 mês. Brasil.

4a) 6 meses

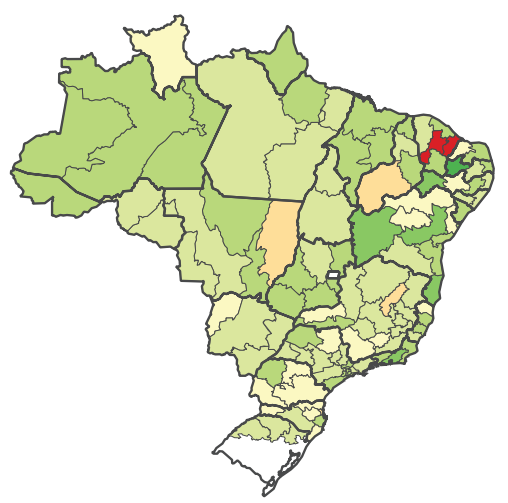

4b) 3 meses

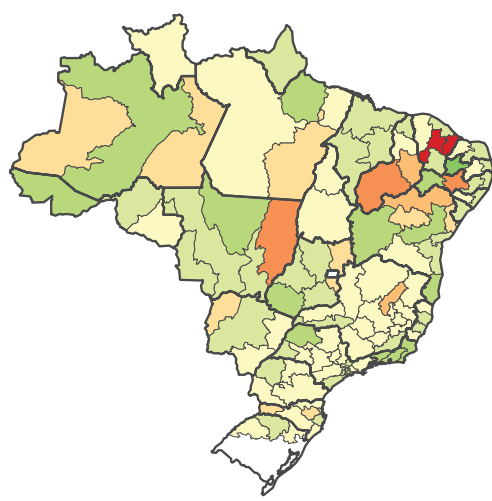

4c) 1 mês

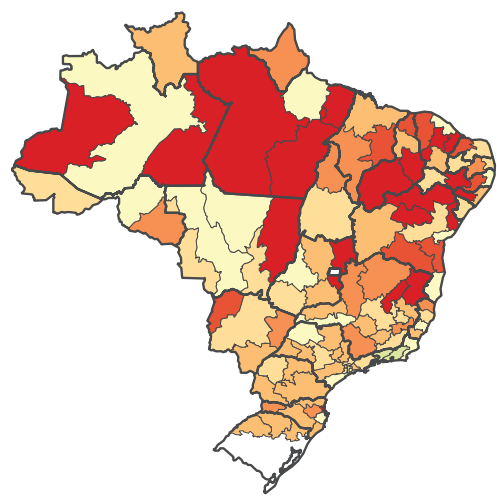

$\%$ $0-20$ $20-40$ $40-60$ $60-80$ 80-100 $100-120$ 120-140

140-160 $160-180$

$180-200$

$200+$ 
aquelas com casos, as taxas de infecção corrigidas variavam entre 0,007\% e 2,3\%. Aproximadamente $49 \%$ das microrregiões tinham taxas inferiores a $0,1 \%$, já $3 \%$ experimentavam taxas maiores que $1 \%$, localizadas principalmente no Amazonas e Amapá.

Analisando a pressão da demanda sobre leitos gerais (Figuras 5a, 5b e 5c), 20 microrregiões, localizadas no Amazonas (5), Amapá (2), Tocantins (1), Maranhão (1), Ceará (3), Sergipe (2) e São Paulo (6), estariam operando com a capacidade de atendimento comprometida se as taxas de infecção alcançassem o valor observado em apenas 1 mês. Considerando um período maior, 3 ou 6 meses, apenas 2 microrregiões vivenciariam esse colapso.

Para leitos de UTI (Figuras 5d, 5e e 5f), se as taxas de infecção atingissem o patamar observado em 1 mês, o colapso do sistema de saúde seria observado em $55 \%$ das macrorregiões. Essa situação seria mais crítica em 18 macrorregiões, onde a demanda superaria em $50 \%$ a oferta existente. Dentre elas, 7 estariam no Norte e 6 no Nordeste. Considerando o cenário de 6 meses, a proporção de macrorregiões com a capacidade de atendimento comprometida de leitos de UTI seria menor (10\%), com apenas 3 macrorregiões apresentando demanda excedente maior que 50\%. Os resultados para aparelhos de ventilação mecânica são similares aos estimados para leitos de UTI (Figuras 5g, 5h e 5i).

\section{Figura 5}

Percentual de leitos gerais e de unidade de terapia intensiva (UTI) e aparelhos de ventilação mecânica ocupados, considerando taxas de infecção por COVID-19 observadas (corrigidas por sub-registros) em cada micro/macrorregião de saúde e três horizontes temporais: 6 meses,

3 meses e 1 mês. Brasil.

5a) Oferta SUS e privada - leitos gerais (microrregião) -6 meses

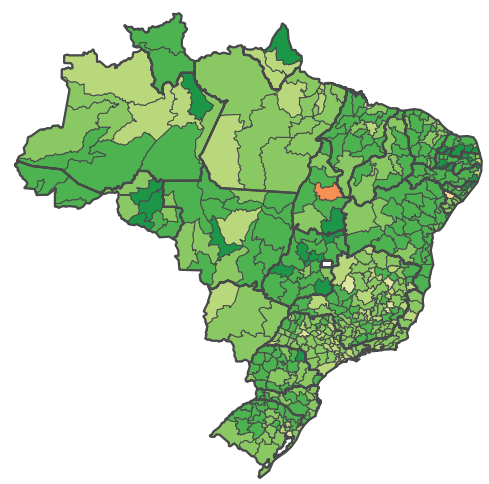

5d) Oferta SUS e privada - leitos de UTI (macrorregião) -6 meses

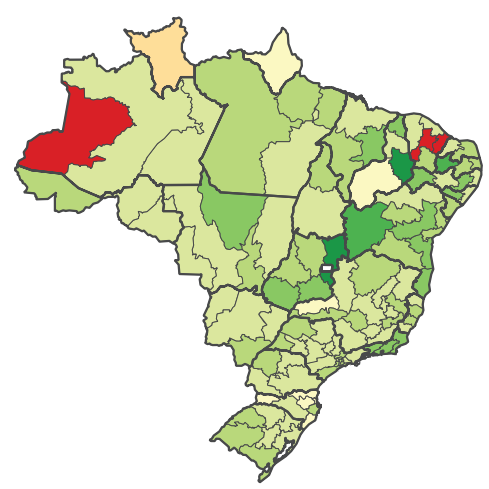

5b) Oferta SUS e privada - leitos gerais (microrregião) -3 meses

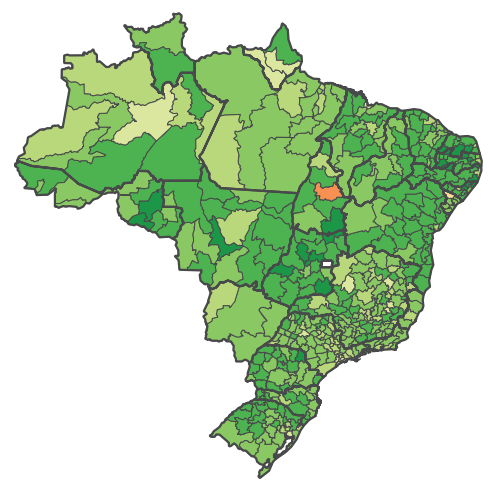

5e) Oferta SUS e privada - leitos de UTI (macrorregião) - 3 meses

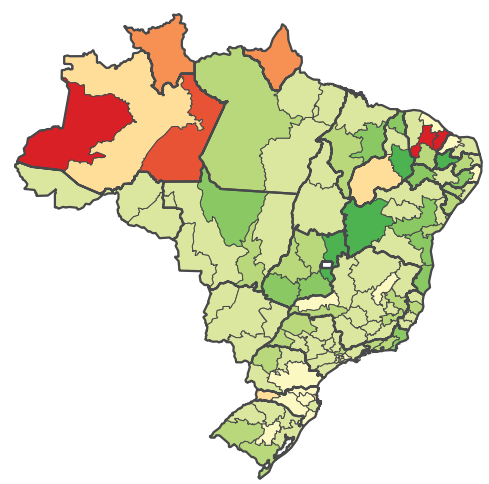

5c) Oferta SUS e privada - leitos gerais (microrregião) - 1 mês

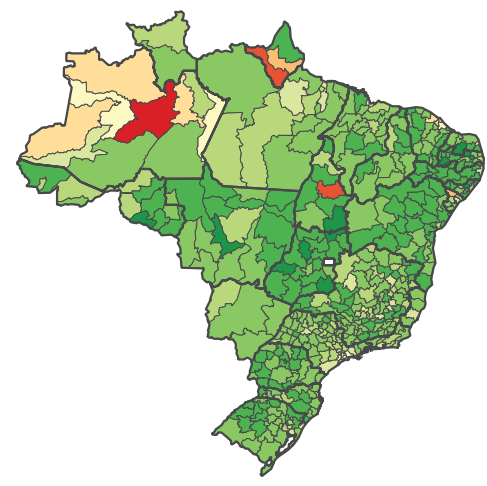

5f) Oferta SUS e privada - leitos de UTI (macrorregião) - 1 mês

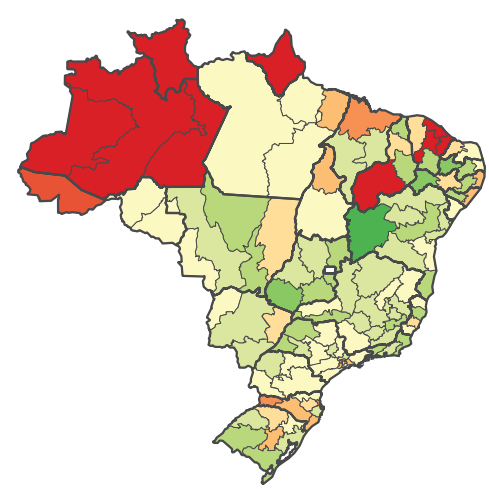

$\%$

0-20

20-40

40-60

60-80

$80-100$

100-120

120-140

140-160

160-180

180-200

$200+$

(continua) 
5g) Oferta SUS e privada - aparelhos de ventilação mecânica (macrorregião) - 6 meses

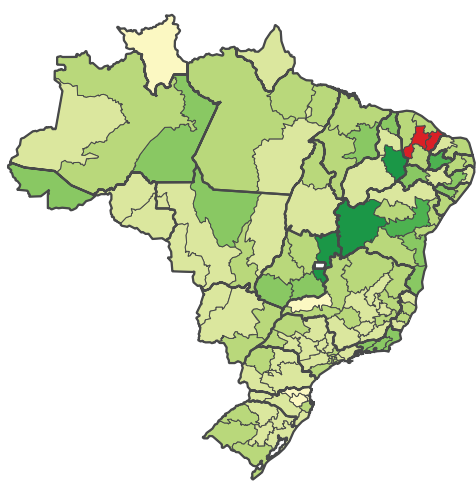

5h) Oferta SUS e privada - aparelhos de ventilação mecânica (macrorregião) - 3 meses

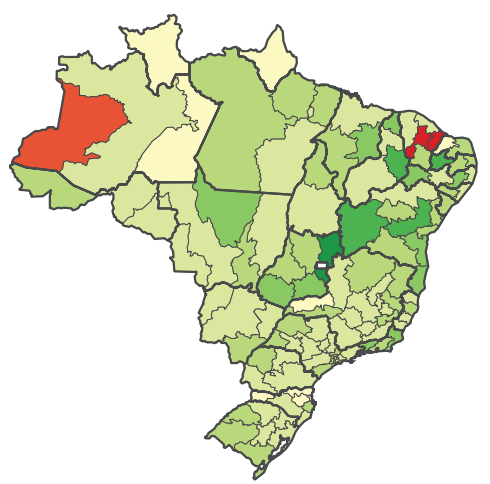

5i) Oferta SUS e privada - aparelhos de ventilação mecânica (macrorregião) - 1 mês

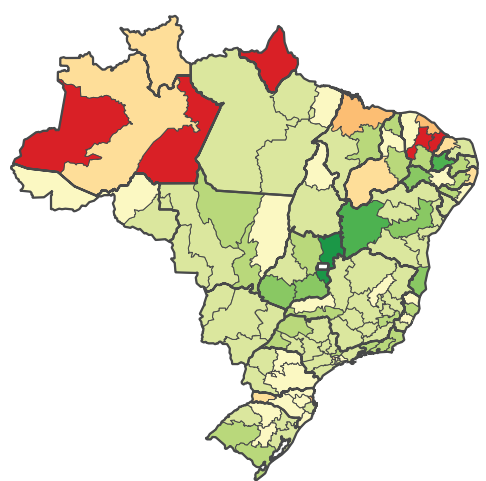

\section{Discussão}

Os resultados encontrados evidenciam uma situação crítica do sistema de saúde para atender à demanda potencial gerada pela pandemia da COVID-19. Essa situação é preocupante porque resulta em aumento da mortalidade nos locais em que a oferta dos serviços não está preparada. Contabilizando a oferta pública e privada, diversas microrregiões e macrorregiões de saúde operariam além de sua capacidade, comprometendo o atendimento principalmente a pacientes com sintomas mais severos. O cenário é pior para leitos de UTI e no Norte e Nordeste do país. A presença de vazios assistenciais pode levar o sistema ao colapso, mesmo com taxas menos elevadas de infecção. Apesar dos problemas de oferta detectados, a propagação mais tardia da COVID-19 no interior do Brasil cria uma janela de oportunidade importante para a reorganização do sistema de saúde local e adoção de medidas de mitigação da propagação da infecção. Ressalta-se, contudo, que para algumas localidades no Norte e Nordeste a propagação do vírus já é uma realidade e vem ocorrendo de forma relativamente acelerada, sofrendo pressão também do lado da demanda, como em Manaus e Fortaleza 25. Nossas simulações realizadas com base nas taxas de infecção observadas até o dia 4 de maio confirmam a fragilidade da oferta dessas duas regiões, associada a uma propagação acelerada da doença. No Sudeste, apesar da maior disponibilidade de oferta, as taxas e velocidade de infecção em algumas localidades estão maiores 25. Em São Paulo e no Rio de Janeiro, além da pressão da demanda, uma questão importante é o papel do setor privado, crucial para a ampliação da oferta.

O estudo apresenta três mensagens relevantes para o sistema de saúde no contexto da pandemia. A primeira refere-se à necessidade de se reduzir a velocidade de propagação da COVID-19 na população brasileira. Conter a propagação será fundamental para aliviar a pressão sobre o sistema de saúde e permitirá um tempo maior para a reorganização da oferta. Dada a heterogeneidade regional, tanto em relação à oferta como em relação às taxas de infecção, não será possível adotar uma única forma de contenção da propagação do vírus no Brasil. Diferentes medidas de contenção estão sendo implantadas no cenário internacional. A forma como vêm sendo adotadas é muito variada entre os países, dependendo do estágio e velocidade de propagação da doença, especificidades do sistema de saúde, além de aspectos sociais, econômicos e políticos 26. De acordo com Canabarro et al. 27, as medidas já implantadas em algumas cidades e estados brasileiros, como cancelamento de aulas em todos os níveis, distanciamento social e quarentena voluntária, têm contribuído para reduzir o número de casos de infecção e postergação do pico de contágio. Devido a questões econômicas, há uma pressão para a suspensão dessas medidas. Se esse processo não for feito de forma gradual e coordenada, consi- 
derando as especificidades de cada município e região, os riscos para a saúde podem ser catastróficos. Deve-se avaliar a capacidade de atendimento do sistema de saúde em cada localidade, monitorar a taxa básica de reprodução do vírus e ampliar os testes laboratoriais para o rastreamento e isolamento dos casos confirmados 27,28 . O teste em massa permite a implantação de uma estratégia mais otimizada de mitigação da propagação da doença, evitando ações mais drásticas que teriam consequências mais graves sobre o setor produtivo 28 . Além disso, sem essas informações é difícil dimensionar a demanda em cada localidade para adequar a oferta. Devido às dificuldades que países de dimensão continental como o Brasil enfrentam, a testagem plena não seria uma opção factível. No entanto, uma cobertura mais elevada poderia ajudar a reduzir a velocidade de propagação e as consequências econômicas para o país. Comparativamente a outros países, o total de testagem no Brasil é ainda muito baixo 26,29.

A segunda mensagem diz respeito à necessidade de se expandir os leitos disponíveis. O setor privado contribui para amortecer o déficit de demanda, mas em várias macrorregiões, dependendo da velocidade com que a infecção se propaga, a oferta conjunta dos dois setores não seria suficiente. Nesse cenário, algumas medidas podem contribuir para ampliar a oferta dos serviços hospitalares, além da colaboração com o setor privado. A construção imediata de hospitais de campanha é necessária e deve vir acompanhada de uma política de alocação de profissionais de saúde e insumos adequados, principalmente onde a oferta é incipiente. A ampliação da oferta já vem sendo verificada em diversas localidades no Brasil. Até o dia 20 de abril, de acordo com o CNES, foram organizados 80 hospitais de campanha, resultando em um incremento de cerca 6.300 leitos. Nessa expansão da oferta, o papel do Governo Federal é fundamental principalmente devido a três aspectos: normatização de uma regra única para relação público/privado; economias de escala e ganhos de barganha na compra de insumos; provimento direto em regiões que não têm capacidade de alavancar esta oferta de forma imediata.

Para o dimensionamento da oferta no longo prazo é necessário ainda considerar o fluxo de demanda usual do sistema, na medida em que há um limite temporal para a postergação de internações eletivas de forma a não comprometer a saúde desses pacientes. Em nossas simulações, descontamos os leitos que são utilizados para o tratamento de outras morbidades, conforme a taxa de ocupação de 2019. Esse procedimento é importante uma vez que não podemos assumir que a demanda por outras causas deixará de ocorrer ou de serem atendidas devido a COVID-19.

É importante ressaltar ainda o papel dos hospitais de pequeno porte, que representam aproximadamente $66 \%$ dos estabelecimentos hospitalares no Brasil. Esses hospitais operam a uma taxa de ocupação muito baixa (26\%), em contraposição à dos hospitais de maior porte (75\%). Existe, portanto, uma capacidade ociosa no sistema hospitalar, mas ela está presente com maior intensidade em hospitais com menor resolutividade 30 . Os hospitais de pequeno porte não estão preparados para o tratamento de pacientes com sintomas mais severos da COVID-19 devido ao alto grau de especialização dos recursos necessários. Dessa forma, a expansão da oferta via hospitais de pequeno porte poderá ser limitada.

A terceira mensagem faz referência à própria organização da oferta dos serviços de saúde que é regionalizada. Em situações de demanda usual, esse desenho é adequado devido à presença de economias de escala. Mas em situações de pandemia, esse desenho pode não ser adequado se a distância que o paciente tiver de percorrer for muito alta dada a rapidez da evolução da doença. Este trabalho mostra que para $8 \%$ dos municípios brasileiros a distância média percorrida para obter atendimento de UTI é superior a $240 \mathrm{~km}$. As maiores distâncias são percorridas na Regiões Norte, com destaque para o Amazonas e Amapá. Dessa forma, é necessário organizar o encaminhamento dos pacientes em condições e tempo adequados para conter a evolução da doença. Isso dependerá, de um lado, da oferta adequada de meios de transporte (terrestres ou aéreos) e, de outro, da capacidade dos municípios de referência da micro/macrorregião em absorver essa demanda. Nesse contexto, ter um sistema de regulação dos leitos dinâmico para otimizar e coordenar o encaminhamento da demanda dentro de cada região será crucial. Devido à rapidez com que os casos graves evoluem, é fundamental que se organize a lógica do referenciamento para evitar tempo de espera que pode levar pacientes ao óbito. O problema maior é quando a própria macrorregião de saúde está deficitária em termos de leitos, conforme detectado nos exercícios de simulação que realizamos.

As estimativas geradas por nossas simulações apresentam pelo menos três limitações. A primeira é o uso dos parâmetros internacionais para estimar a demanda por internações. Nesse caso, estamos supondo que as taxas específicas por idade de internações hospitalares gerais e de UTI por COVID-19 
no Brasil serão iguais às norte-americanas ou espanholas, tanto em relação ao seu nível quanto em relação à sua estrutura por idade. Embora existam informações sobre as taxas de infecção e hospitalização por grupo etário para o Brasil, a cobertura ainda é baixa. Análises preliminares indicam uma similaridade na distribuição de taxas de infecção por idade no Brasil em relação aos Estados Unidos. O caso espanhol apresenta taxas relativamente mais elevadas e mais envelhecidas (Material Suplementar; http://cadernos.ensp.fiocruz.br/static/arquivo/suppl-e00115320-pt_4059.pdf). Desse modo, como a desagregação por grupo etário é um diferencial metodológico deste trabalho, optamos por manter os parâmetros internacionais focando a análise das estimativas baseadas no caso norte-americano.

A segunda limitação é referente à heterogeneidade espacial das taxas de morbidade que podem afetar os desfechos de internação por COVID-19 em cada microrregião 31. Em certa medida, parte dessa variação foi captada em nosso estudo por meio da distribuição da população por idade. Entretanto, mesmo considerando que a transição de saúde e demográfica seja correlacionada, podem restar variações regionais na morbidade dentro dos grupos de idade.

Finalmente, nosso estudo não leva em consideração as necessidades de profissionais diretamente envolvidos na assistência aos pacientes, tampouco aqueles envolvidos no suporte e higienização que são essenciais para o funcionamento adequado dos hospitais na resposta à pandemia. Apesar dessas limitações, nossos resultados fornecem um diagnóstico importante da situação de oferta no Brasil no início da pandemia e em que medida a propagação do vírus vem afetando a capacidade de atendimento do sistema de saúde em cada localidade.

\section{Colaboradores}

K. V. M. S. Noronha, G. R. Guedes, C. M. Turra e M. V. Andrade participaram de todas as etapas de execução do trabalho, desde a concepção, desenho do estudo, organização dos bancos de dados, estimação dos resultados até a escrita e revisão do manuscrito. L. Botega, D. Nogueira e L. Carvalho participaram da organização dos bancos de dados, estimação dos resultados, bem como da escrita e revisão do manuscrito. J. A. Calazans participou da escrita, revisão do manuscrito, organização do banco de dados e estimação dos resultados. L. Servo e M. F. Ferreira participaram da escrita e revisão do manuscrito.

\section{Informações adicionais}

ORCID: Kenya Valeria Micaela de Souza Noronha (0000-0002-7174-6710); Gilvan Ramalho Guedes (0000-0001-8231-238X); Cássio Maldonado Turra (0000-0003-4051-3567); Mônica Viegas Andrade (0000-0002-6821-1598); Laura Botega (0000-00016030-4100); Daniel Nogueira (0000-0002-32067782); Julia Almeida Calazans (0000-0002-62153251); Lucas Carvalho (0000-0002-3618-3967); Luciana Servo (0000-0003-0770-7378); Monique Félix Ferreira (0000-0003-2513-4382).

\section{Agradecimentos}

O presente trabalho foi realizado com o apoio da Coordenação de Aperfeiçoamento de Pessoal de Nível Superior (CAPES; código de financiamento 001). K.V.M.S.N., G.R.G., M.V.A. e C.M.T. agradecem ao Conselho Nacional de Desenvolvimento Científico e Tecnológico (CNPq) pelo desenvolvimento deste estudo, por meio da concessão da bolsa de produtividade. J.A.C. também agradece à mesma entidade pela bolsa de doutorado. Todos os autores agradecem os comentários de Pedro Amaral e o auxílio técnico de Jeferson Andrade, ambos da Universidade Federal de Minas Gerais (UFMG). 


\section{Referências}

1. World Health Organization. Oxygen sources and distribution for COVID-19 treatment centres: interim guidance, 4 April 2020. Geneva: World Health Organization; 2020.

2. Sarkar J, Chakrabarti P. A machine learning model reveals older age and delayed hospitalization as predictors of mortality in patients with COVID-19. medRxiv 2020; 30 mar. https://www.medrxiv.org/content/10.1101/2 020.03.25.20043331v1.

3. Zhang T, McFarlane K, Vallon J, Yang L, Xie J, Blanchet J, et al. A model to estimate bed demand for COVID-19 related hospitalization. medRxiv 2020; 26 mar. https://www.medrxiv. org/content/10.1101/2020.03.24.20042762v1.

4. IHME COVID-19 Health Service Utilization Forecasting Team; Murray C. Forecasting COVID-19 impact on hospital bed-days, ICUdays, ventilator-days and deaths by US state in the next 4 months. medRxiv 2020; 30 mar. https://www.medrxiv.org/content/10.1101/2 020.03.27.20043752v1.

5. Waldman A, Shaw A, Ngu A, Campbell S. Are hospitals near me ready for coronavirus? Here are nine different scenarios. ProPublica 2020; 17 mar. https://projects.propublica.org/ graphics/covid-hospitals.

6. Moghadas SM, Shoukat A, Fitzpatrick MC, Wells CR, Sah P, Pandey A, et al. Projecting hospital utilization during the COVID-19 outbreaks in the United States. Proc Natl Acad Sci U S A 2020; 117:9122-6.

7. Verhagen MD, Brazel DM, Dowd JB, Kashnitsky I, Mills M. Predicting peak hospital demand: demographics, spatial variation, and the risk of "hospital deserts" during COVID-19 in England and Wales, 2020. OSFPreprints 2020; 27 mar. https://osf.io/g8s96/.

8. Walker PGT, Whittaker C, Watson O, Baguelin M, Ainslie KEC, Bhatia S, et al. The global impact of COVID-19 and strategies for mitigation and suppression. https://spiral.imperial. ac.uk:8443/handle/10044/1/77735 (acessado em 31/Mar/2020).

9. CDC COVID-19 Response Team. Severe outcomes among patients with coronavirus disease 2019 (COVID-19). MMWR Morb Mortal Wkly Rep 2020; 69:343-6.

10. Petrilli CM, Jones SA, Yang J, Rajagopalan H, O'Donnell LF, Chernyak Y, et al. Factors associated with hospitalization and critical illness among 4,103 patients with COVID-19 disease in New York City. medRxiv 2020; 11 abr. https://www.medrxiv.org/content/10.110 1/2020.04.08.20057794v1.

11. Rentsch CT, Kidwai-Khan F, Tate JP, Park LS, King Jr. JT, Skanderson M, et al. Covid-19 testing, hospital admission, and intensive care among 2,026,227 United States veterans aged 54-75 years. medRxiv 2020; 14 abr. https:// www.medrxiv.org/content/10.1101/2020.04. $09.20059964 \mathrm{v} 1$
12. Castro MC, Carvalho LR, Chin T, Kahn R, Franca G, Macario EM, et al. Demand for hospitalization services for COVID-19 patients in Brazil. medRxiv 2020; 1 abr. https://www.me drxiv.org/content/10.1101/2020.03.30.20047 $662 \mathrm{v} 1$.

13. Rache B, Nunes L, Rocha R, Lago M, Fraga A. Como conter a curva no Brasil? Onde a epidemiologia e a economia se encontram. São Paulo: Instituto de Estudos para Políticas de Saúde; 2020. (Nota Técnica, 4).

14. Rache B, Rocha R, Nunes L, Spinola P, Massuda A. Para além do custeio: necessidades de investimento em leitos de UTI no SUS sob diferentes cenários da Covid-19. São Paulo: Instituto de Estudos para Políticas de Saúde; 2020. (Nota Técnica, 7).

15. Departamento de Economia e Estatística, Secretaria de Planejamento, Orçamento e Gestão. Coronavírus: exercícios de projeções de casos para o Rio Grande do Sul. https://colunado nene.com.br/wp-content/uploads/2020/03/ Coronavirus-Exerc\%C3\%ADcio-de-Proje\% C3\%A7\%C3\%A3o-de-casos-no-Rio-Grandedo-Sul.pdf (acessado em 19/Mar/2020).

16. Batista A, Antunes B, Faveret G, Peres I, Dantas L, Bastos L, et al. Dimensionamento de leitos para os casos de infecção por COVID-19 no Estado do Rio de Janeiro para o dia 04 de abril de 2020. Rio de Janeiro: Núcleo de Operações e Inteligência em Saúde; 2020. (Nota Técnica, 2).

17. Batista A, Antunes B, Faveret G, Peres I, Dantas L, Bastos L, et al. Dimensionamento de leitos para os casos de infecção por COVID-19 nas Regiões de Saúde do Sudeste de Minas Gerais (Juiz de Fora e região) para o dia 09 de abril de 2020. Rio de Janeiro: Núcleo de Operações e Inteligência em Saúde; 2020. (Nota Técnica, 2.1).

18. Noronha K, Guedes G, Turra CM, Andrade MV, Botega L, Nogueira D, et al. Pandemia por COVID-19 em Minas Gerais, Brasil: análise da demanda e da oferta de leitos e equipamentos de ventilação assistida considerando os diferenciais de estrutura etária, perfil etário de infecção, risco etário de internação e distâncias territoriais. São Paulo: Instituto de Estudos para Politicas de Saúde; 2020. (Working Paper Series, 4).

19. Freire FHMA. Projeção populacional municipal com estimadores bayesianos, Brasil 2010 2030. In: Sawyer DO, coordenador. Seguridade social municipal. Projeto Brasil 3 Tempos. Brasília: Secretaria Especial de Assuntos Estratégicos da Presidência da República/Programa das Nações Unidas para o Desenvolvimento/ Centro Internacional de Políticas para o Crescimento Inclusivo; 2019. 
20. Dirección General de Salud Pública, Calidad e Innovación, Secretaria General de Sanidad, Ministerio de Sanidad. Actualización no 85. Enfermedad por el coronavirus (COVID-19). 24.04.2020 (datos consolidados a las 21:00 horas del 23.04.2020). https://www.mscbs.gob. es/profesionales/saludPublica/ccayes/alerta sActual/nCov-China/documentos/Actuali zacion_85_COVID-19.pdf (acessado em 27/ Abr/2020).

21. Ferguson NM, Laydon D, Nedjati-Gilani G, Imai N, Ainslie K, Baguelin M, et al. Impact of non-pharmaceutical interventions (NPIs) to reduce COVID-19 mortality and healthcare demand. London: Imperial College COVID-19 Response Team; 2020.

22. Ribeiro LC, Bernardes AT. Estimate of underreporting of COVID-19 in Brazil by acute respiratory syndrome hospitalization reports. Belo Horizonte: Centro de Desenvolvimento e Planejamento Regional, Universidade Federal de Minas Gerais; 2020. (Nota Técnica).

23. Carvalho LR. Distribuição espacial da oferta de saúde no Brasil no contexto do mix público-privado [Dissertação de Mestrado]. Belo Horizonte: Universidade Federal de Minas Gerais; 2017.

24. Organisation for Economic Cooperation and Development. Health at a glance 2019: OECD indicators. Paris: OECD Publishing; 2019.

25. Ministério da Saúde. Situação epidemiológica: doença pelo coronavírus 2019. Boletim COE COVID-19 2020; (13). https://portalarqui vos.saude.gov.br/images/pdf/2020/April/21/ BE13---Boletim-do-COE.pdf.

26. Pescarini J, Aquino E, Silveira I, Aquino R, Souza-Filho J. Medidas de distanciamento social no controle da pandemia de COVID-19: potenciais impactos e desafios no Brasil. SciELO Preprints 2020; 20 abr. https://preprints.scie lo.org/index.php/scielo/preprint/view/116/ version/121.
27. Canabarro A, Tenorio E, Martins R, Martins L, Brito S, Chaves R. Data-driven study of the COVID-19 pandemic via age-structured modelling and prediction of the health system failure in Brazil amid diverse intervention strategies. medRxiv 2020; 15 abr. https://www.me drxiv.org/content/10.1101/2020.04.03.20052 498v2.

28. Barreto ML, Barros AJD, Carvalho MS, Codeço CT, Hallal PRC, Medronho RA, et al. O que é urgente e necessário para subsidiar as políticas de enfrentamento da pandemia de COVID-19 no Brasil? Rev Bras Epidemiol 2020; 23:e200032.

29. Global Business Data Platform. Rate of coronavirus (COVID-19) tests performed in the most impacted countries worldwide as of May 25, 2020 (per million population). https://www. statista.com/statistics/1104645/covid 19 testing-rate-select-countries-worldwide/ (acessado em 05/Mai/2020).

30. Botega LA, Andrade MV, Guedes GR. Brazilian hospitals' performance: an assessment of the Unified Health System (SUS). Health Care Manag Sci 2020; [Online ahead of print].

31. Borges GM. Health transition in Brazil: regional variations and divergence/convergence in mortality. Cad Saúde Pública 2017; 33:e00080316. 


\section{Abstract}

This study aims to analyze the pressure on the Brazilian health system from the additional demand created by COVID-19. The authors performed a series of simulations to estimate the demand for hospital beds (health micro-regions) as well as to ICU beds, and mechanical ventilators (health macro-regions) under different scenarios of intensity (infection rates equivalent to 0.01, 0.1, and 1 case por 100 inhabitants) and time horizons (1, 3, and 6 months). The results reveal a critical situation in the system for meeting this potential demand, with numerous health micro-regions and macro-regions operating beyond their capacity, compromising the care for patients, especially those with more severe symptoms. The study presents three relevant messages. First, it is necessary to slow the spread of COVID-19 in the Brazilian population, allowing more time for the reorganization of the supply and relieve the pressure on the health system. Second, the expansion of the number of available beds will be the key. Even if the private sector helps offset the deficit, the combined supply from the two sectors (public and private) would be insufficient in various macro-regions. The construction of field hospitals is important, both in places with a history of "hospital deserts" and in those already pressured by demand. The third message involves the regionalized organization of health services, whose design may be adequate in situations of routine demand, but which suffer additional challenges during pandemics, especially if patients have to travel long distances to receive care.

COVID-19; Pandemics; Health Services Accessibility; Unified Health System; Supplemental Health

\section{Resumen}

El objetivo de este estudio es analizar la presión sobre el sistema de salud brasileño, ocasionada por la demanda adicional de camas hospitalarias y equipos de ventilación mecánica, generada por el COVID-19. Para tal fin, se realizó un conjunto de simulaciones, con el fin de estimar la demanda de camas generales (microrregiones de salud), camas de UTI y equipamientos de ventilación asistida (macrorregiones de salud) en diferentes escenarios, según la intensidad (tasas de infección equivalentes a 0,01, 0, 1 y 1 caso por 100 habitantes) y horizontes temporales (1, 3 y 6 meses). Los resultados evidencian una situación crítica del sistema para atender esa demanda potencial, ya que diversas microrregiones y macrorregiones de salud operarían más allá de su capacidad, comprometiendo la atención a pacientes principalmente aquellos con los sintomas más graves. El estudio presenta tres mensajes relevantes. En primer lugar, es necesario reducir la velocidad de propagación del COVID-19 en la población brasileña, permitiendo un tiempo mayor para la reorganización de la oferta y aliviando la presión sobre el sistema de salud. En segundo lugar, es necesario expandir el número de camas disponibles. A pesar de que el sector privado contribuya a amortiguar el déficit de demanda, la oferta conjunta de los dos sectores no sería suficiente en varias macrorregiones. La construcción de hospitales de campaña es importante, tanto en lugares donde históricamente existen lagunas asistenciales, como también en aquellos donde ya se observa una presión por parte de la demanda. El tercer mensaje se refiere a la organización por regiones de los servicios de salud que, a pesar de ser adecuada en situaciones de demanda habitual, en momentos de pandemia, este diseño implica desafíos adicionales, especialmente si la distancia que el paciente tuviera que recorrer fuera muy lejana.

COVID-19; Pandemias; Accesibilidad a los Servicios de Salud; Sistema Único de Salud; Salud Complementaria
Recebido em 07/Mai/2020

Versão final reapresentada em 11/Mai/2020

Aprovado em 12/Mai/2020 